\title{
Exploring foods that can make protein claims in terms of best choices from food groups
}

In Ireland, food based dietary guidelines (FBDG) are developed to reduce obesity, cardiovascular disease and cancer ${ }^{(1)}$. Food portions providing key nutrients that are lower in energy and saturated fat and higher in fibre, are recommended using food groups. Consumers are more attracted by foods bearing claims, particularly in relation to protein ${ }^{(2)}$. The aims of this study are to (a) explore recommended portions of foods within four food groups (Vegetables, Cereals, Dairy, Meat \& Alternatives) to identify those that best provide key nutrients, and $(b)$ assess which foods can bear protein claims.

Recommended adult portion sizes for 213 foods identified from FBDG for $1-5$ year olds ( $n 134)$ and the general population ( $n 79)$, were grouped according to the four food groups. Glycaemic load (GL) for Vegetable and Cereal foods (where available), iron, DHA and EPA content of Meat \& Alternative foods were assessed. For each food, the number of nutrition claims and whether 'a source of' or 'high in' protein claims could be made was assessed. Foods were ranked as higher quality according to a low ratio of energy:fibre (for Vegetables and Cereals Groups), a high ratio of calcium:saturated fat (SFA) for Dairy Group) and of protein:SFA (for Meat \& Alternatives Group). Higher and lower quality foods (lowest $50 \%$ on ranking) were compared using non-parametric tests in SPSS (version 25).

The higher $v s$. lower quality foods in the Vegetables Group were significantly lower in energy and GL, higher in fibre and could bear more claims; in the Cereals Group they were higher in fibre and could bear more claims; in the Dairy Group they were higher in calcium, lower in saturated fat and could bear less claims; in the Meat \& Alternatives Group they were lower in energy and saturated fat, and comparable in terms of claims. High proportions of foods could bear claims to be 'a source of' or 'high in' protein in the Dairy (98\% and $84 \%$, respectively) and in the Meat \& Alternatives ( $98 \%$ and $93 \%$, respectively) Groups. Approximately half of foods in the Vegetables Group could claim to be 'a source of' protein (55\%) and 41\% 'high in' protein; whereas $48 \%$ of foods in the Cereals Group could claim to be 'a source of' protein and $2 \%$ to be 'high in' protein.

Higher quality foods can be identified by ranking according to key nutrients and these foods can generally bear more nutrition claims. Despite having a low protein quality, a high proportion of foods in the Vegetables and Cereals Groups can bear protein claims, demonstrating that such claims do not take account of protein quality. In conclusion, this study indicates that protein quality should be included in the conditions of use for protein claims.

1. Healthy Ireland Food Pyramid (2016).

2. Fernan, Schuldt \& Niederdeppe (2018) Heath Commun 33, 12, 1425-1433. 